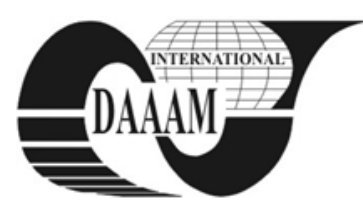

Annals of DAAAM for 2011 \& Proceedings of the 22nd International DAAAM Symposium, Volume 22, No. 1, ISSN 1726-9679 ISBN 978-3-901509-83-4, Editor B. Katalinic, Published by DAAAM International, Vienna, Austria, EU, 2011 Make Harmony between Technology and Nature, and Your Mind will Fly Free as a Bird Annals \& Proceedings of DAAAM International 2011

\title{
STRUCTURAL AND FUNCTIONAL OPTIMIZATION OF INDUSTRIAL ROBOT'S NC AXES
}

\author{
AVRAM, G[eorgia] - C[ezara]; NICOLESCU, A[drian] F[lorin]; STRAJESCU, E[ugen] R[adu] \& PETRE, \\ M[arian]
}

\begin{abstract}
The paper presents the original contributions on the numerical controlled (NC) axes' structural and functional optimization of industrial robots $(I R)$ with electric drive system. The main purpose of the paper is to define and validate an assisted selection procedure of IR's NC axes' optimal structure. A complex assisted study on NC axes' optimal structure of a GUDEL gantry robot for part handling application has been performed for this purpose. IR's NC axes specific performance parameters and their influences on the robot's overall performances were also studied. The study's results show the conformity of procedure, the real robot design and the manner the real robot design / overall performances may be improved. Key words: industrial robots, numerically controlled axes, electric driving systems, optimal design, software modelling
\end{abstract}

\section{INTRODUCTION}

The structural and functional optimization of each IR's NC axes is essential for increasing the overall performance of the IR, as the structure and individual performances of each axis, influence IR's overall performances. This optimization should be done accordingly the application particularities the robot is incorporated in. There is a complete range of the IR overall performance features covering all of the IR constructive types currently operational and respectively, classes of applications they can be incorporated in. In accordance with the particularities of the robotic application, the IR should be evaluated only by those performance criteria that are specific to the robotic applications for which the IR is used, selected from ir's overall performance characteristics specified in ISO 9283 $(* * *, 1996)$.

\section{GENERALIZED ALGORITHM FOR ELECTRIC DRIVEN NC AXIS OPTIMAL COMPONENT SELECTION}

The type of the robotic application that incorporates the IR is critical when it comes to design its electro-mechanical structure. To define the optimal structure and sizing of the IR's electromechanical systems components, the following elements should be taken into consideration (Fig. 1) (Collins, 2009; Voss, 2007): Load - the magnitude, direction and sense of the resistant forces applied to the driven element of a NC axis; Orientation of the motion axis, the movement plane and potential obstacles in the movement plane; Speed, acceleration and deceleration along with inertia forces applied to the moving element of the NC axis; Travel - stroke, stroke length, actuation elements; Accuracy - accuracy in positioning and repeatability in the final index position; Environmental - ambient temperature, lubrication conditions, presence of corrosive agents; Work cycle - the ratio of non - operating and operating time and influence of non - thermal effects on the static and dynamic behaviour of the IR's components and assemblies.

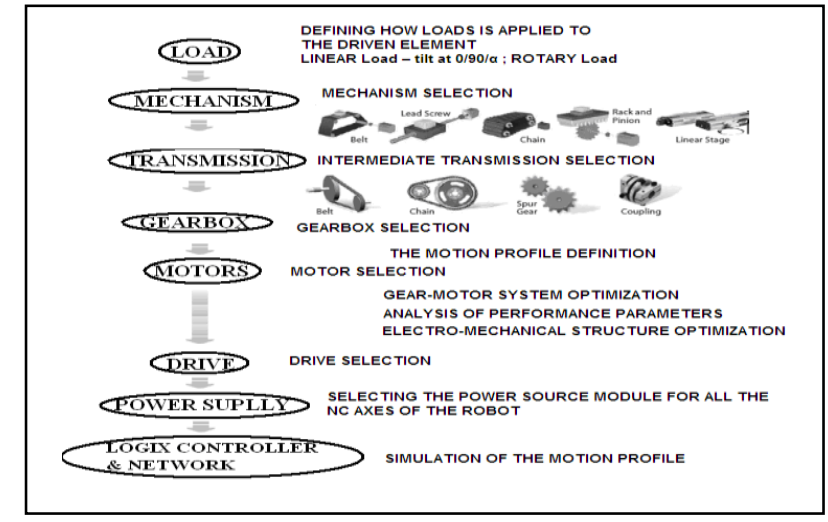

Fig.1. Organizational chart

\section{STRUCTURAL AND FUNCTIONAL OPTIMIZATION OF NC AXES OF A GANTRY ROBOT WITH ELECTRIC DRIVING SYSTEMS}

As subject of analysis, a GUDEL FP4 gantry robot was selected (Fig. 2) (***, 2010).

The study was conducted taking into account the manufacturer's specifications regarding IR's specific design and performances characteristics and according some IR's physical characteristics evaluated by mean of its virtual prototype (achieved in CATIA V5), as is the case for the weight of IR's assemblies moving along $\mathrm{X}, \mathrm{Y}, \mathrm{Z}$ linear axes. The performed study allowed the following achievements (Avram, 2011):

- Emphasizing the importance of a systematic approach on structural and functional optimization of the IRs' NC axes.

- Identifying the functional limitations of predefined $\mathrm{NC}$ axes structures (considering existing design of the studied IR), by analysing the design and functional characteristics of the mechanical components and of the electric drive systems of each IR's NC axis.

- Drawing conclusions on the possibility to improve NC axis / IR's overall functional parameters by redesigning existing NC axes' structure / resizing driving systems of the studied IR.

The specific objectives for improving NC axis / IR's overall functional parameters / performances refer to:

- Performance characteristics of the NC axes determined for different motion profiles. Focus on the of the mobile elements speed stability and optimizing necessary torque for driving mobile elements on each axis.

- Keeping in allowed limits for each axis mobile element's positioning accuracy variation.

- Focus on IR's overall performance parameters: identifying workspace areas of optimal exploitation for the studied IR, indicating the acceptable limits of torques / maximum speeds and thermal regimes of the IR servo-drives for different process parameters.

- Recommend necessary IR's design improvement having as result NC axis / overall IR's performances improvement. 


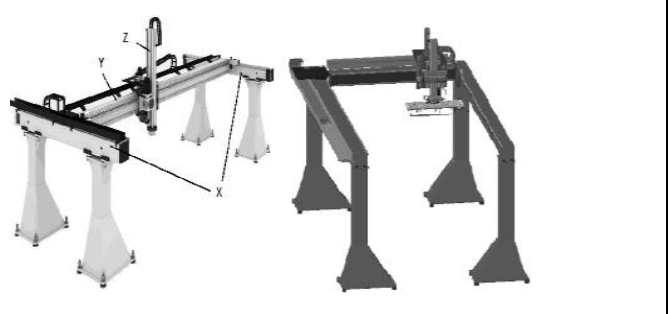

Fig. 2. Virtual prototype of the gantry robot

\section{ASSISTED STRUCTURE AND PERFORMANCE OPTIMIZATION FOR THE STUDIED NC AXES}

When configuring the NC axis of the studied IR, the information provided by the manufacturer was taken into account, in terms of the IR GUDEL FP 4's mechanical structure. In addition, specific issues for testing the electric drive system and identifying a specific NC axis behavior in relation to its overall performance were addressed.

In terms of nominal torque and peak torque, the listed values are as following: to achieve travel speeds up to $37.3 \%$ of maximum speed, it is necessary to use a driving motor supplying a rated torque of $1.58999 \mathrm{Nm}$ and a peak torque of $3.57448 \mathrm{Nm}$; to achieve travel speeds rating from $42.7 \%$ and up to $75 \%$ of the maximum speed it is necessary to use a driving motor supplying a rated torque of $1.60508 \mathrm{Nm}$ and a peak torque of 5.00811 Nm (Fig. 4).

Considering the maximum value of IR's payload, a trapezoidal motion profile and whole range of positioning speed for each $\mathrm{NC}$ axis, the number of operating points at nominal torque diagram (see Fig 3) and following the analysis of the results obtained from the axis performance simulation (see Fig. 5) for the mobile elements positioning error and speed stabilizing, the simulation results diagrams for axis output performances are illustrated in Fig. 6.
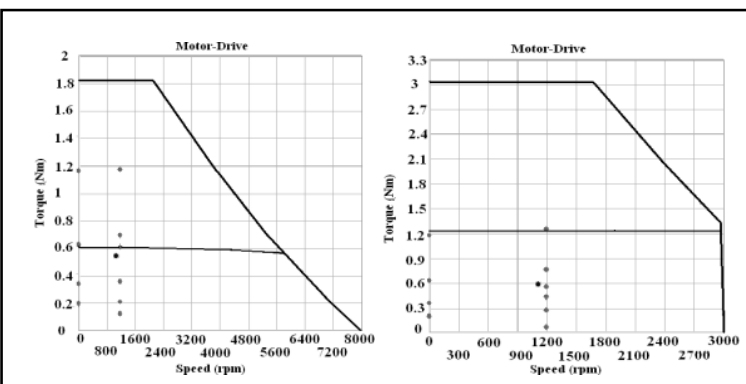

Fig. 3. Speed-Torque Diagram

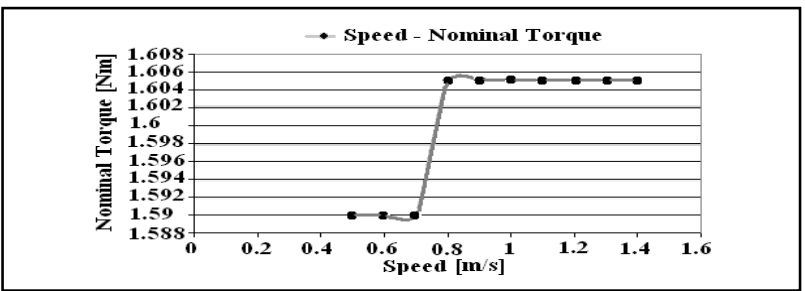

Fig. 4. Speed-Nominal Torque Diagram

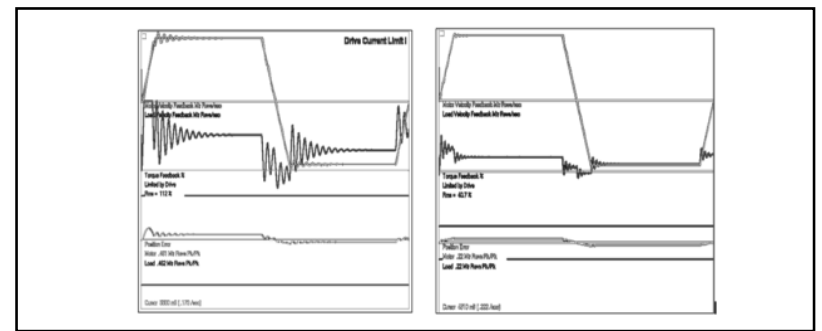

Fig. 5. Results obtained from simulation
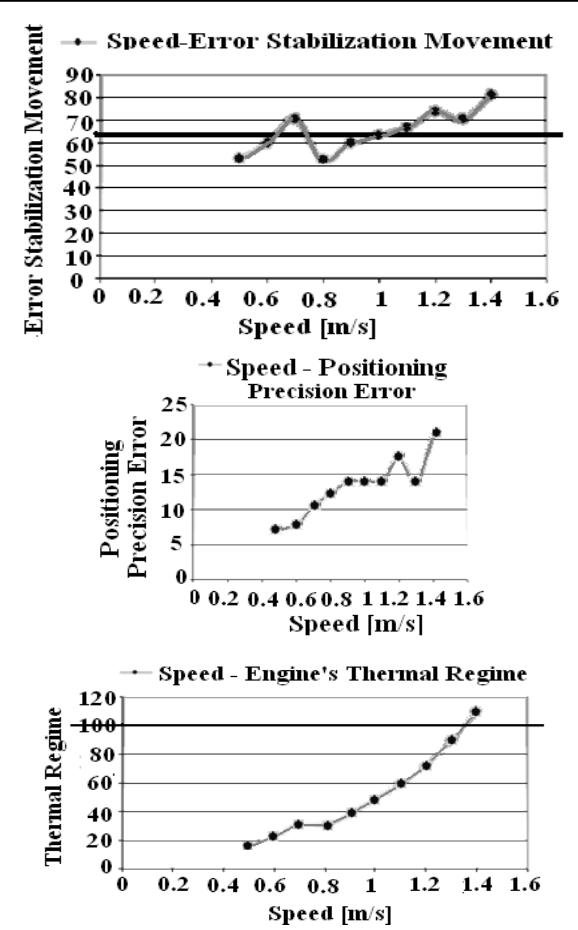

Fig. 6. Results obtained from the axis performance simulation

\section{CONCLUSIONS}

The IR's overall performance analysis showed three ranges of limits: the very good behavior $(<0.5 \mathrm{~m} / \mathrm{s})$, good $(0.5-1 \mathrm{~m} /$ s) and poor $(>1.2 \mathrm{~m} / \mathrm{s})$. In respect to real robot $\mathrm{NC}$ axis electro-mechanical structure and electric drive systems overall performance as indicated by the manufacturer, it was found that, for the constructive solution adopted by the manufacturer, for speeds over $65-75 \%$ of the maximum value IR's performance drastically decreases, contradicting the manufacturer's specifications.

Solutions for $\mathrm{NC}$ axes optimal structure and electrical driving systems of the GUDEL FP 4 robot were identified and can be used to increase individual performance of each $\mathrm{NC}$ axis as well as IR's overall performances.

The NC axis performance analysis was done in quasi-static regime of overall IR's thermal behavior. Specific research will be carried out, to continue current works, considering the influence of IR's thermal behavior in transitory thermal conditions on $\mathrm{NC}$ axis and the IR's overall performances.

\section{REFERENCES}

Avram C. (2011). Structural and functional optimization of NC axes of RI with electric drive systems to increase their overall performance, Dissertation Project, UPB

Collins, D. (2009). Robots in Medical Applications: How LOSTPED and Cartesian Robots Can Help, Available from: http://www.robotics.org/content-detail.cfm/IndustrialRobotics-Feature-Article/Robots-in-Medical-Applications:How-LOSTPED-and-Cartesian-Robots-CanHelp/content_id/1870, Accessed: 2011-01-14

Voss, W. (2007). A Comprehensible Guide to Servo Motor Sizing, Copperhill Technologies Corporation, ISBN 978-09765116-1-8, United States of America

*** (1996). International Standard ISO 9283:1996, Manipulating Industrial Robots - Performance criteria and related test methods

*** (2010). GÜDEL Modules Catalog, D, F, E / 07.08 / NR. 0112130, Available from: http://www.gudel.com/modules/, Accessed: 2010-12-10 